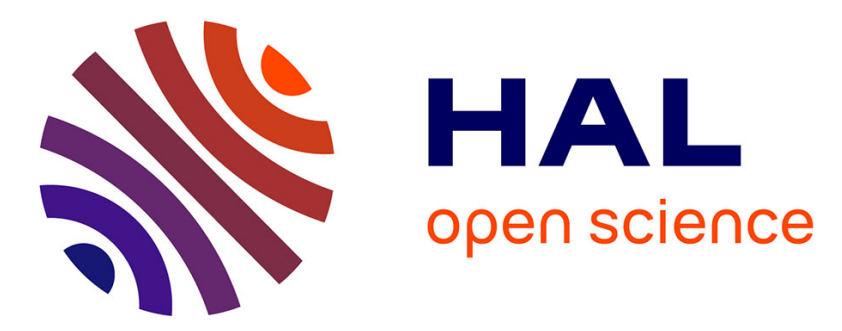

\title{
Fabrication and Magnetic Actuation of 3D-Microprinted Multifunctional Hybrid Microstructures
}

Victor Vieille, Roxane Pétrot, Olivier Stéphan, Guillaume Delattre, Florence Marchi, Marc Verdier, Orphee Cugat, Thibaut Devillers

\section{- To cite this version:}

Victor Vieille, Roxane Pétrot, Olivier Stéphan, Guillaume Delattre, Florence Marchi, et al.. Fabrication and Magnetic Actuation of 3D-Microprinted Multifunctional Hybrid Microstructures. Advanced Materials Technologies, 2020, 5 (10), pp.2000535. 10.1002/admt.202000535 . hal-03048907

\section{HAL Id: hal-03048907 https://hal.science/hal-03048907}

Submitted on 10 Dec 2020

HAL is a multi-disciplinary open access archive for the deposit and dissemination of scientific research documents, whether they are published or not. The documents may come from teaching and research institutions in France or abroad, or from public or private research centers.
L'archive ouverte pluridisciplinaire HAL, est destinée au dépôt et à la diffusion de documents scientifiques de niveau recherche, publiés ou non, émanant des établissements d'enseignement et de recherche français ou étrangers, des laboratoires publics ou privés. 


\title{
Fabrication and Magnetic Actuation of 3D-Microprinted Multifunctional Hybrid Microstructures
}

\author{
Victor Vieille,* Roxane Pétrot, Olivier Stéphan, Guillaume Delattre, Florence Marchi, \\ Marc Verdier, Orphée Cugat, and Thibaut Devillers*
}

Only achievable with two photons' polymerization, 3D printing at the micrometer scale is essential for the fabrication of complex objects such as photonic components, deformable microstructures, or microscaffolds for biological cells. Integrating magnetic materials inside those structures has made their remote actuation with an external magnetic field possible. However, the nature of the magnetic material, its volume, and precise position in the structure are keys for the efficiency, dexterity, and compatibility with optical or biological functions. Herein, an original approach consisting in the bonding of discrete and fully magnetic microbeads to unaffected 3D-microprinted structures is presented. Implemented in combination with the fine control of optical and mechanical properties allowed by the careful design of the 3D architecture, it is applied to the fabrication of the first remotely tunable biconvex microlens (focal length of $18 \mu \mathrm{m}$ ). Combined with the additional precise positioning and magnetic orientation of multiple microbeads, the presented technique enables the fabrication of complex actuators such as a $100 \mu \mathrm{m}$ microtweezer that can be translated, rotated, and opened with a single variable external magnetic field. The dexterity of this untethered micromanipulator is demonstrated through a pick-and-place operation of $40 \mu \mathrm{m}$ objects in a confined environment.

geometry of the printed structures by an external stimulus, as is done in the socalled $4 \mathrm{D}$ printing. ${ }^{[7,8]}$ One of the most promising ways for remote stimulation is magnetic actuation, ${ }^{[9-11]}$ which can be performed accurately and instantaneously in confined environments through a time-varying external magnetic field. Nevertheless, despite recent advances, ${ }^{[12-19]}$ the incorporation of magnetic materials into 3D-microprinted structures remains a major bottleneck for the fabrication of robust, versatile, and dexterous microactuators. ${ }^{[20]}$ Alternative strategies to integrate the magnetic materials will be key ${ }^{[21]}$ to combining the full potential of both 3D microprinting and magnetic actuation. Here, we implemented a new, simple approach consisting of bonding discrete magnetic microbeads to a microprinted undoped soft structure. The individualized localization and orientation of magnetization of each bead prior to bonding allows for various modalities of remote actuation such as displacement, rotation, and controlled deformation. Thanks to the hybrid

Thanks to shape and material versatility, 3D micro/nanoprinting ${ }^{[1]}$ enables unique functions required for a variety of systems such as photonic structures, ${ }^{[2,3]}$ cell scaffolds, ${ }^{[4,5]}$ or microfluidic components, ${ }^{[6]}$ promising significant advances in areas such as biomedicine, data transfer, or imaging sensors. Particularly interesting is the possibility of modifying the

Dr. V. Vieille, R. Pétrot, Dr. F. Marchi, Dr. T. Devillers

Univ. Grenoble Alpes, CNRS, Grenoble INP, Institut Néel

25 rue des martyrs, Grenoble 38000, France

E-mail: victor.vieille@neel.cnrs.fr; thibaut.devillers@neel.cnrs.fr

Dr. V. Vieille, R. Pétrot, Dr. O. Cugat

Univ. Grenoble Alpes, CNRS, Grenoble INP, G2ELab

21 rue des martyrs, Grenoble 38000, France

Dr. O. Stéphan, G. Delattre

Univ. Grenoble Alpes, CNRS, Grenoble INP, LIPhy

140 Rue de la Physique, Saint-Martin-d'Hères, Grenoble 38402, France

Dr. M. Verdier

Univ. Grenoble Alpes, CNRS, Grenoble INP, SIMAP

1130 Rue de la Piscine, Saint-Martin-d'Hères, Grenoble 38402, France nature of the designed systems, it is possible to simultaneously exploit the strong magnetic torques and forces induced on a hard ferromagnetic bead and to benefit from the biocompatibility, deformability, high transparency, precise, and personalized manufacture of the microprinted polymer. Based on this approach, we built the first 3D-printed and magnetically actuated microlens for photonics, and a dexterous microgripper well suited to the complex manipulation of single biological cells. Through these two state-of-the-art examples, we demonstrate the power and versatility of our technique to achieve the association of complex motion with multifunctional capabilities at the micrometric scale.

To design magnetic structures capable of complex motion and strong mechanical work, two critical parameters must be considered for the magnetic material: the magnitude of its magnetization, which will directly affect the intensity of the magnetic response to an external magnetic field, and the possibility of defining locally the direction of the magnetization and maintaining this heterogeneity throughout the whole structure. For these reasons, high-performance permanent magnets, based on $\mathrm{Nd}_{2} \mathrm{Fe}_{14} \mathrm{~B}$ with high remanence and coercivity, are particularly relevant, and have been successfully used in millimeter structures to perform dexterous actuation. ${ }^{[6,22-24]}$ 
Combined with 3D printing, arbitrary complex geometries can be built, which considerably broadens the available mechanical functionalities. ${ }^{[25]}$ Nevertheless, when going to micrometric dimensions, even if dexterous but weak actuation is possible in $2 \mathrm{D},{ }^{[26]}$ it gets more challenging with $3 \mathrm{D}$ printing, for which two-photon polymerization (TPP) is needed. Indeed, during the TPP process, the photoresist needs to remain sufficiently transparent to the laser. Thus, to integrate the opaque magnetic material into the printed object, three approaches have been reported: the dilution of magnetic nanoparticles in the photoresist, ${ }^{[15-17,27]}$ the deposition of thin magnetic films or particles after printing and washing, ${ }^{[5,12-14]}$ or more complex and indirect, the filling of a printed mold. ${ }^{[28-30]}$

In some of these approaches, the small amount or low performance of the embedded magnetic material considerably limits the magnitude of the forces and torques achievable. Furthermore, the difficulty to control locally the orientation of the magnetization reduces critically the potential dexterity of the devices, as the whole structure undergoes the same magnetic stimulation. Moreover, the alteration of the initial photoresist impairs some functional characteristics such as transparency ${ }^{[31]}$ prohibiting any kind of microprinted optics based on magnetically doped polymers. To tackle these issues, we propose a unique approach consisting of bonding a regular transparent TPP structure to fully dense hard ferromagnetic beads, in order to manufacture versatile, robust, and dexterous magnetic microactuators in the $10-100 \mu \mathrm{m}$ range.
Figure 1 and Videos S1 and S2 (Supporting Information) show the successive steps to build a simple hybrid structure by what we call direct laser bonding (DLB). In this first approach, the designed microstructure is constructed by TPP with a UV-curable polymer and bonded to a spherical polycrystalline $\mathrm{NdFeB}$ bead ( $\approx 25 \mu \mathrm{m}$ diameter). To avoid the complexity of prepositioning precisely the beads on the substrate, they are randomly distributed in the photoresist, and the direct laser writing (DLW) objects are constructed close to given beads, with undoped photoresist (Figure 1A). Thanks to the optical resolution of the microscope, the relative positioning of the structure with respect to the bead can be achieved with a precision of around $1 \mu \mathrm{m}$. To bond the bead to the finished 3D object, the laser goes from the DLW object to the bead, curing the photoresist along the way (Figure 1B,C). The link is finalized when the focal point of the laser reaches the surface of the bead, resulting in a strong adhesion between the polymer structure and the bead (Figure 1D). It is worth mentioning that when the focal point of the laser gets close to the bead, the metallic material absorbs light through the one-photon classical absorption process, resulting in local heating (although not high enough to thermally demagnetize the bead) and the formation of bubbles in the resin, which are resorbed within a few tens of seconds. Therefore, it is preferable to build a broad link with a higher laser power rather than scanning back and forth, which would require to wait until the bubbles are resorbed before the next pass. The optimal area to create the link is near the mid-height of the bead, where the geometry

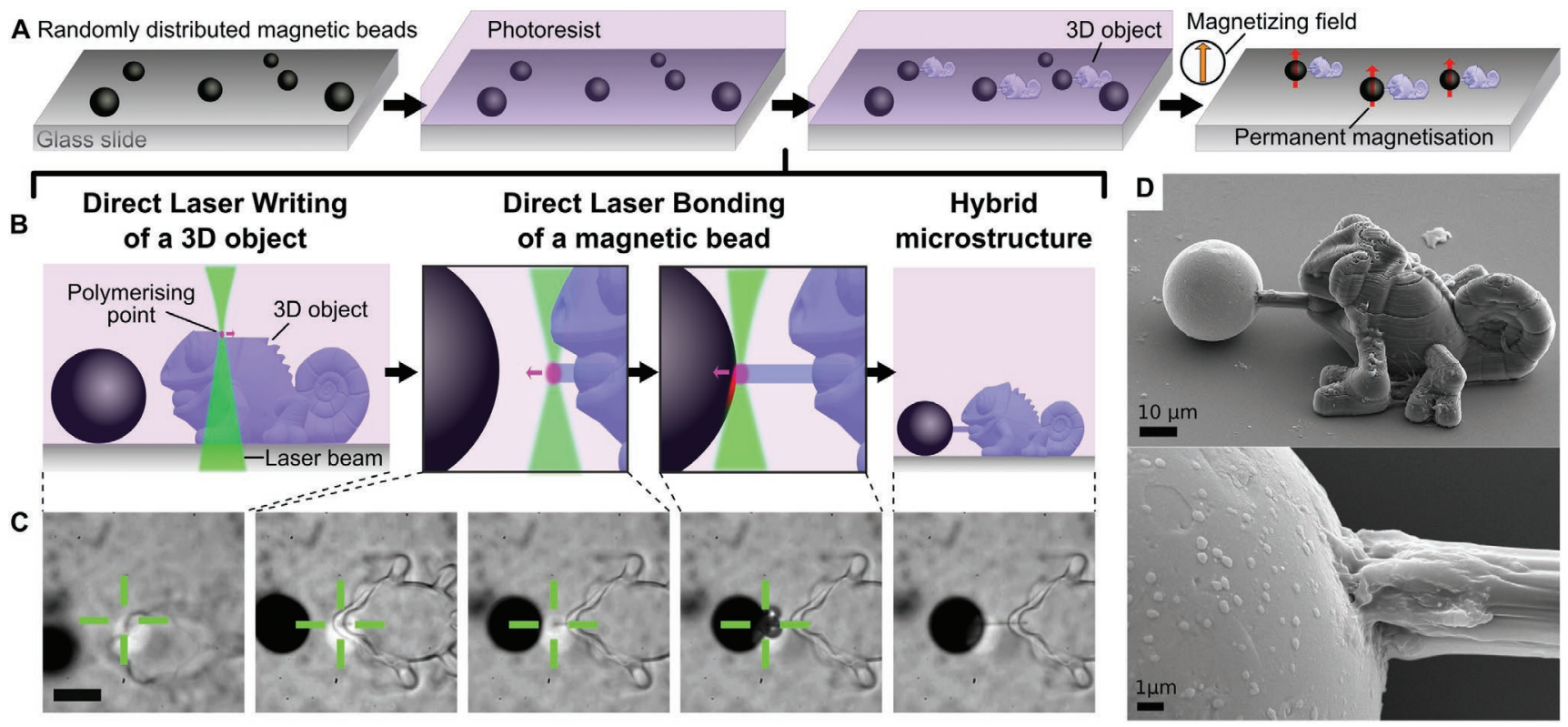

Figure 1. TPP fabrication of a one-bead microstructure. A) General process flow: magnetic beads are randomly dispersed on a glass slide and covered with photoresist. The microstructure is then built near a bead by direct laser writing (DLW), then bonded to the bead. Lastly, the slide is washed in solvent to eliminate the uncured photoresist and unbound beads. A magnetic field is then applied to magnetize the beads in the desired direction. B) Schematics of the bonding details. First, the 3D object is fabricated using the DLW technique. Direct laser bonding (DLB) is then performed as follows: a link is made by DLW between the object and the bead at the mid-height of the bead. In addition, when the laser illuminates the bead, onephoton absorption occurs, which locally heats the photoresist. C) Optical microscopy images of the corresponding fabrication steps for a structure composed of a NdFeB bead and a 3D object (Video S1, Supporting Information). Two bubbles appear briefly due to one-photon absorption heating. The green cross represents the laser spot, which is invisible because of optical filters. D) Scanning electron microscopy (SEM) image of the hybrid structure and close-up on the adhesion area. 
allows the photoresist to be properly cross-linked in close contact with the bead itself (Figure S1, Supporting Information). To evaluate the order of magnitude of the mechanical tensile strength of adhesion, we designed a test based on hybrid structures (Figure S2, Supporting Information). The measured adhesive resistance is in the range of $10^{6} \mathrm{~N} \mathrm{~m}^{-2}$ that is enough to survive washing steps and magnetic actuation. Multiple links were also developed to address the cases requiring greater adhesion.

Once the microstructures are printed, bound to the magnetic bead and washed, the beads are magnetized by an external magnetic field of typically $0.5 \mathrm{~T}$ (Figure S3, Supporting Information). The remanent magnetization can be tuned by the applied magnetic field, and the choice of magnetically isotropic and highly coercive $\mathrm{NdFeB}$ beads enables their magnetization in any arbitrary direction. Despite the magnetization being far from saturated, the magnetic fields used for subsequent actuation are small enough $(<100 \mathrm{mT})$ not to modify it, so that the magnetization of the bead can be considered as constant to a first approximation.

Subject to an external magnetic field and in order to minimize the magnetostatic energy $E=-V * \vec{M} \cdot \vec{B}$ ( $V$ is the volume, $\vec{M}$ is the magnetization, and $\vec{B}$ is the external magnetic field), the bead undergoes a torque ${ }^{[32]}$ proportional to $\vec{M} \times \vec{B}$, which tends to align its magnetization with the external field and a force proportional to the magnetic field gradient. The magnetic torque is not only the driving force for the actuation of our structures, but it is also a key component in the fabrication process of more advanced multibead actuators by enabling the precise and individual positioning and magnetic orientation of every single bead.

In Figure 2 (and Video S3, Supporting Information), we demonstrate the fabrication of such a complex structure. In this approach, the beads are again randomly distributed in the liquid polymer. The body of the microactuator is first produced by TPP and fixed to the glass slide in standard conditions. By applying a rotating magnetic field of low magnitude $(\approx 10 \mathrm{mT})$, we produce a rotational motion of beads which, thanks to mechanical friction with the glass slide below, induces the bead to roll in the desired direction (Figure 2B). A given bead can thus be placed at a precise location $( \pm 2 \mu \mathrm{m})$, and its magnetization direction ultimately aligned with the external magnetic field. The bead is then bonded to the structure by DLB, so that it can no longer move. These steps are repeated for every bead required in the hybrid structure, the other free beads dispersed in the solution moving accordingly. This method enables the programming of a heterogeneous magnetization pattern in a micrometer scale TPP structure in such a way that the $3 \mathrm{D}$ geometry and magnetization pattern are independent of each other, contrary to prior work. ${ }^{[19]}$ In addition, the photoresist properties such as transparency and elasticity are preserved.

These two methods increase actuation predictability, intensity, and dexterity, while preserving the functionalities of the DLW structure. Moreover, they allow rapid prototyping, as it needs no complex process development. Therefore, we were able to develop novel microdevices never before fabricated at this scale for application fields as diverse as photonics and cell biology.
Photonics is a field in which shape- and material-based functions provided by TPP are particularly relevant. For instance, the TPP fabrication of micro-optical elements used for imaging or coupling integrated photonic components was recently demonstrated. ${ }^{[2,3]}$ We fabricated a complex magneto-mechano-optical 3D structure (Figure 3) based on the first technique presented (Figure 1). It is composed of three main functional elements (Figure 3A): a transparent microlens used to form an optical image, the hinge allowing the rotation of the structure and thus the lens's vertical displacement, and the magnetic bead enabling remote magnetic torque actuation under an external magnetic field. The difference in functionality between the lens and the hinge comes only from the shape difference achieved via TPP. The microlens has a focal length of $18 \mu \mathrm{m}$. To demonstrate the active focusing capabilities of this microsystem, it was placed above an inverted optical microscope in such a way that the microscope objective is focused on the microlens itself. A millimeter-size drawing was positioned above the lens (Figure 3B). The application of a rotating magnetic field induces the rotation of the arm around the hinge, which displaces the lens over a range of about $20 \mu \mathrm{m}$, and thus focuses the image in the observation area of the objective (Figure 3C; Video S4 and Figure S4, Supporting Information). In principle, since the focal length is comparable to the displacement range, this type of lens can be used for fast focusing objects at distances from $100 \mu \mathrm{m}$ to infinity. This simple proof of concept illustrates how powerful TPP is when combined with magnetic bead actuation and its potential applications in remotely actuated microphotonics, for example, attached to the tip of an optical fiber for endoscopic applications, or directly constructed on a light source or detector.

The technique described in Figure 2 was used to fabricate a highly dexterous untethered microgripper inspired from the literature, ${ }^{[22]}$ at a scale ten times smaller and with a $3 \mathrm{D}$ shape (Figure 2A). The key idea of this untethered gripper is that the beads, which are magnetized in different directions, do not undergo the same torque when exposed to an external magnetic field. Thus, the beads can have different functions depending on their magnetic orientation (Video S5, Supporting Information). As shown in Figure 4A, the beads on the gripper arms are magnetized perpendicularly to the gripper's long axis, in opposite directions. On the contrary, the bead on the head is magnetized parallel to the long axis and is therefore is not compensated. Thus, when a magnetic field is applied along the gripper axis, the torque on the head bead is zero, while the torques induced in the arms compensate each other; they have the same magnitude in opposite directions, so the gripper does not move around. However, the arms can open and close due to the individual torques on the arms beads (Figure S5, Supporting Information), depending on the magnitude of the magnetic field. Moreover, as the single bead on the head is not compensated, it tends to align with the external magnetic field, allowing orientation control. Continuous rotation of the structure leads to rolling thanks to friction with the substrate, including gripper displacement by what we call "rolling displacement." The microgripper can also move by translation thanks to the magnetic gradients, as it will slide up the gradient, what we call "gradient displacement." Combining the controlled deformation of the gripper with rolling and gradient 

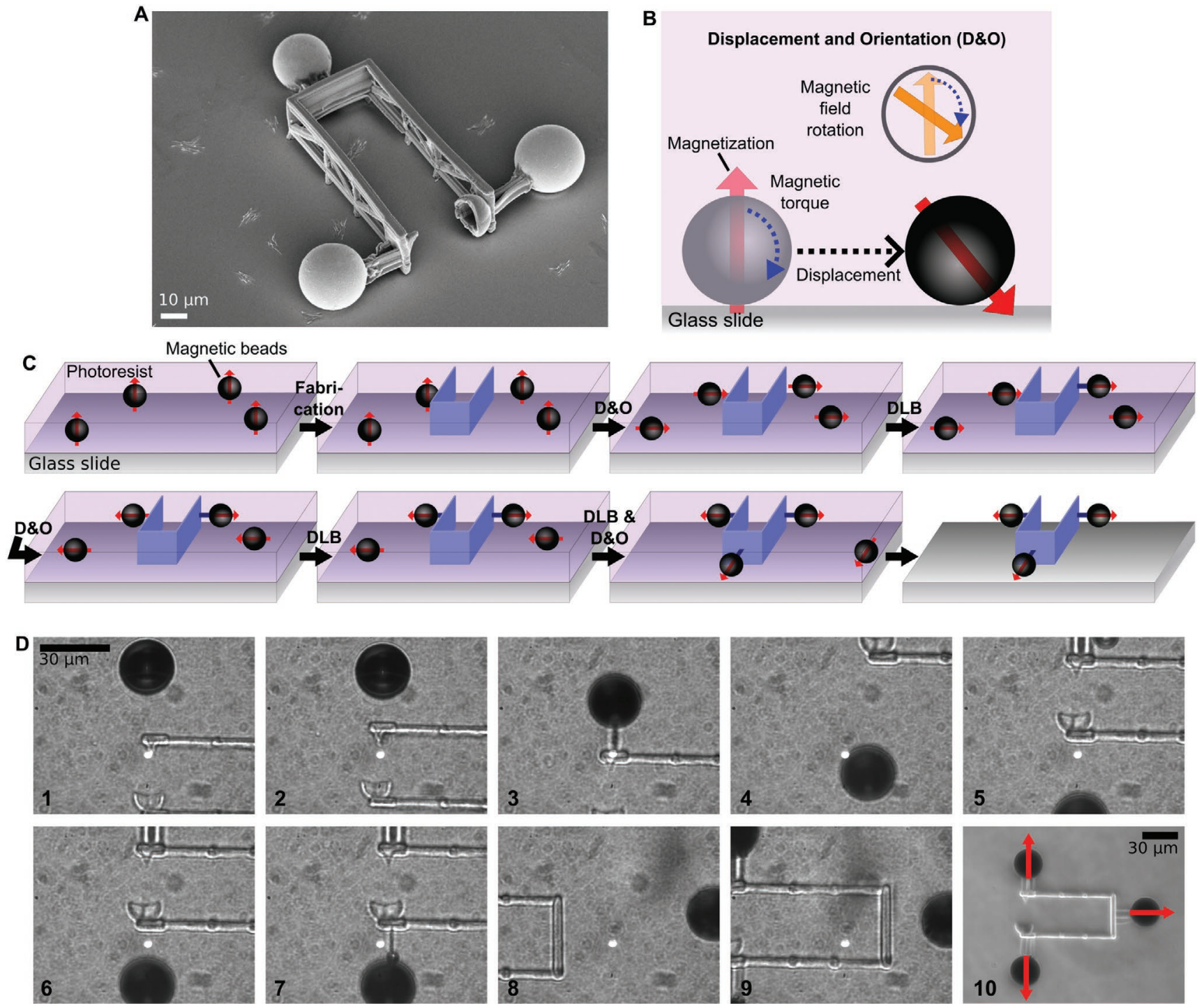

Figure 2. Fabrication of a multibead microstructure. A) SEM image of a microgripper with punching tool (scale bar: $10 \mu \mathrm{m}$ ). B) Schematic of the positioning of a bead by displacement and orientation (D\&O). Under the magnetic torque induced by the external magnetic field, the bead rotates and rolls due to friction with the glass slide. C) Fabrication process (Video S3, Supporting Information). Magnetic beads are randomly dispersed on a glass slide, covered with photoresist, and magnetized. Next, a gripper is built by DLW at a preset place in a region with appropriately distributed beads. A rotating magnetic field is then applied to bring the bead to the appropriate location (gripper is fixed to the glass, but all free beads move together). The first bead is bonded to the gripper by direct laser bonding (DLB). The same process is repeated for binding the remaining beads. Finally, the slide is washed in a solvent, to eliminate the photoresist and the unbound beads. D) Optical microscopy image of the gripper fabrication. The beads are individually moved to the relevant location and bonded to the TPP structure.

displacements, we successfully manipulated polystyrene beads of $40 \mu \mathrm{m}$ diameter (Figure 4B,C; Video S6, Supporting Information) thus demonstrating the possibility of remotely picking and placing micrometric objects in a confined environment. In addition, custom tools can easily be 3D-printed on the gripper, such as a punching tool (Figure 2A). This could prove useful to carry out microsurgery or various experiments on single cells, for instance, cell sorting, mechanical stimulation, force measurement, or enucleation.

The process that we demonstrated is generic and can be applied to a wide range of both polymer and magnetic materials. Although the results presented here were achieved with an Ormocomp-based mixture, we also demonstrated (see the "Experimental Section" and Video S2 in the Supporting Information) the fabrication and magnetic actuation of a collagen-based microprinted structure. This hybrid material will considerably extend the capabilities of magnetic hydrogels, which are particularly relevant for drug delivery ${ }^{[2]}$ and cell scaffolding. ${ }^{[33]}$

The strength of the demonstrated process resides in the fact that despite its apparent simplicity, it is an enabling technology. This technique is the only one to allow at the same time a real 3D geometry, sizes in the $100 \mu \mathrm{m}$ range with a resolution better than $1 \mu \mathrm{m}$ and the possibility of designing a heterogeneous magnetization pattern essential for dexterous actuation. In the following, we will discuss how the presented microsystems 

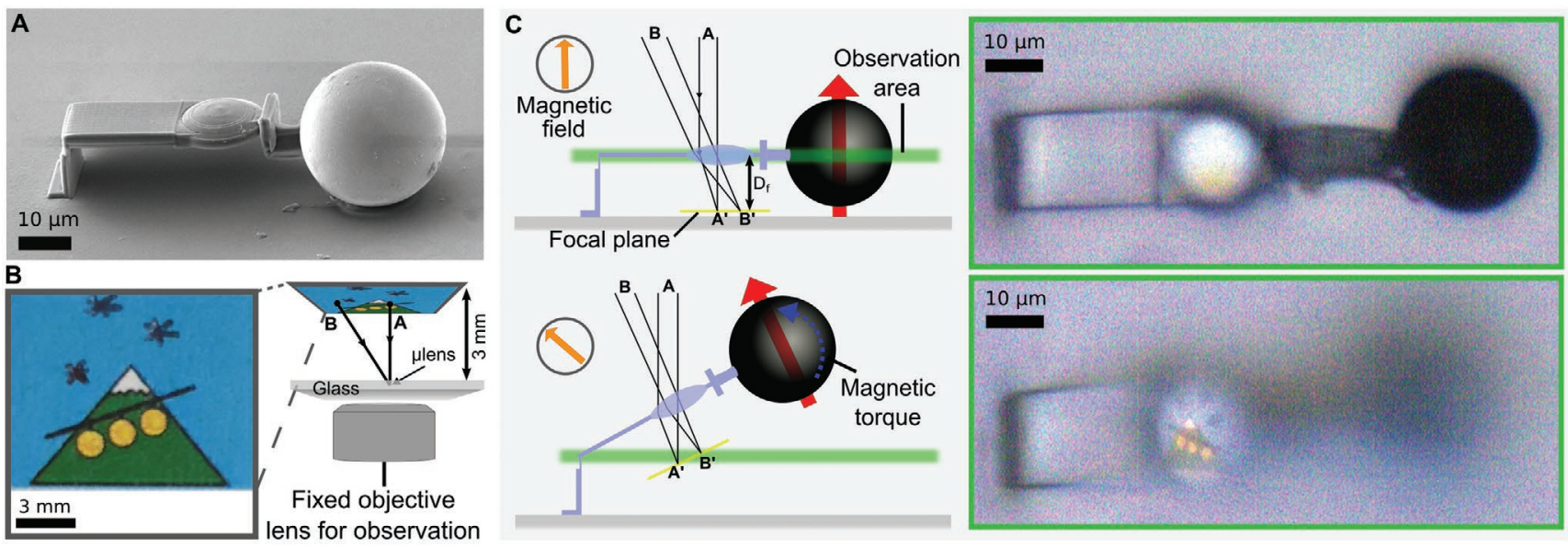

Figure 3. Magnetically actuated hybrid micrometric lens. A) SEM image of the microlens supported by a glass slide. B) Imaging setup. The glass slide supporting the microlens is placed to produce an image of the lens itself, which is sharp and centered on the CCD of the microscope. The optical object (drawing) is positioned $3 \mathrm{~mm}$ above the microlens, so that it can be considered at infinity and illuminated from top. C) Magnetic actuation of the microlens for image focusing. The base of the structure is fixed to the glass slide. A thinner deformable section creates a hinge between the base and the moving part. The magnetic bead is permanently magnetized in the upward direction, and is bonded to the 3D-printed object. The photoresist part of the structure is transparent to visible light $(n=1.52)$. The microlens is a biconvex lens with a focal length $D_{\mathrm{f}}=18.3 \mu \mathrm{m}$. When the magnetic field rotates, it exerts a magnetic torque on the bead, which makes the structure (including the microlens) move around its hinge. The focal plane of the lens also moves, and the image can thus be brought to the object plane of the microscope by magnetic actuation. Right: optical images showing the lens at rest (top) and the image of the drawing (bottom).

compare to previously reported microrobots from state-of-the art literature.

To achieve complex motion of a magnetically actuated robot through bending or articulation, it is essential to design a heterogeneous magnetization pattern that allows for heterogeneous forces and torques in the structure. Several techniques were developed over the last 10 years to perform this magnetic patterning on $3 \mathrm{D}$ structures at the centimeter $\mathrm{scal}^{[25]}$ or on $2 \mathrm{D}$ structures at the millimeter scale,,$^{[6,22,24,34]}$ and at the submillimeter scale. ${ }^{[26,35]}$ What we demonstrate here is that the combination of TPP with magnetic bead positioning and magnetization orientation allows us to reach for the first time a heterogeneous magnetization pattern at length scales at least ten times smaller than before, with the freedom to build an arbitrary complex 3D deformable structure. The resulting great dexterity at dimensions close to typical cell sizes opens wide prospects for cell manipulation and assembly.

Furthermore, thanks to the high magnetic anisotropy induced by the magnetocrystalline anisotropy of $\mathrm{NdFeB}$, it becomes possible to design robots of arbitrary geometries, which was impossible with systems based on super-paramagnetic or magnetically soft materials, for which the magnetic anisotropy originates from shape anisotropy. In addition to the decoupling between magnetic anisotropy and the shape of the structure, the use of a hard magnetic material makes its magnetization relatively independent of the applied magnetic actuation field, allowing for higher torques at very low magnetic field, as well as preventing magnetization reversal at higher fields. A broad range of magnetic field can therefore be used, thus extending the range of forces and torques that can be generated.

This effect is reinforced by the significantly higher amount of magnetic material incorporated in our structures compared with previous works dealing with TPP. When magnetic nanoparticles are embedded directly in the $\operatorname{resin}^{[15-17,27]}$ the volume fraction cannot exceed $4 \%$ to maintain the transparency of the resin, which is necessary for the printing process. When a magnetic film is deposited by physical vapor deposition on top of a TPP structure, ${ }^{[5,13,14]}$ the thickness of magnetic material is in the $100 \mathrm{~nm}$ range which is still relatively small compared to the overall structure. By using gas-atomized NdFeB beads that are fully dense, we achieve structures where the amount of magnetic material can reach $50 \%$ of the total volume, with the printed part still transparent and suitable for optical purposes. By doing this, we achieved the first magnetically activated microoptical device Finally, the technique developed is relatively accessible and easy to use. Two-photon lithography aside, the materials used are easily available, do not require any complex preparation and are of almost negligible costs. Therefore, and considering the fast dissemination of the TPP technology, this process is light and affordable, and has the potential to become a standard tool for the fabrication of magnetic microactuators.

It has to be noted that in the structures presented here, the beads were only partially magnetized in the stray field of a bulk $\mathrm{NdFeB}$ magnet and are still far from saturation (Figure S3, Supporting Information). One order of magnitude could be gained by saturating the magnetization of the beads in a stronger field. Moreover, the magnetic actuation so far performed with a hand-held magnet will be considerably improved with a 3D-magnetic field generator based on multiple electromagnets and 3D-nested coils, resulting in a higher accuracy and reproducibility of the actuator motion. This automated actuation of magnetic beads combined with optical bead recognition will also be used to perform the fabrication in a more automated and reliable way.

The introduction of magnetically hard NdFeB microbeads in TPP microstructures is enabling the fabrication of powerful and 

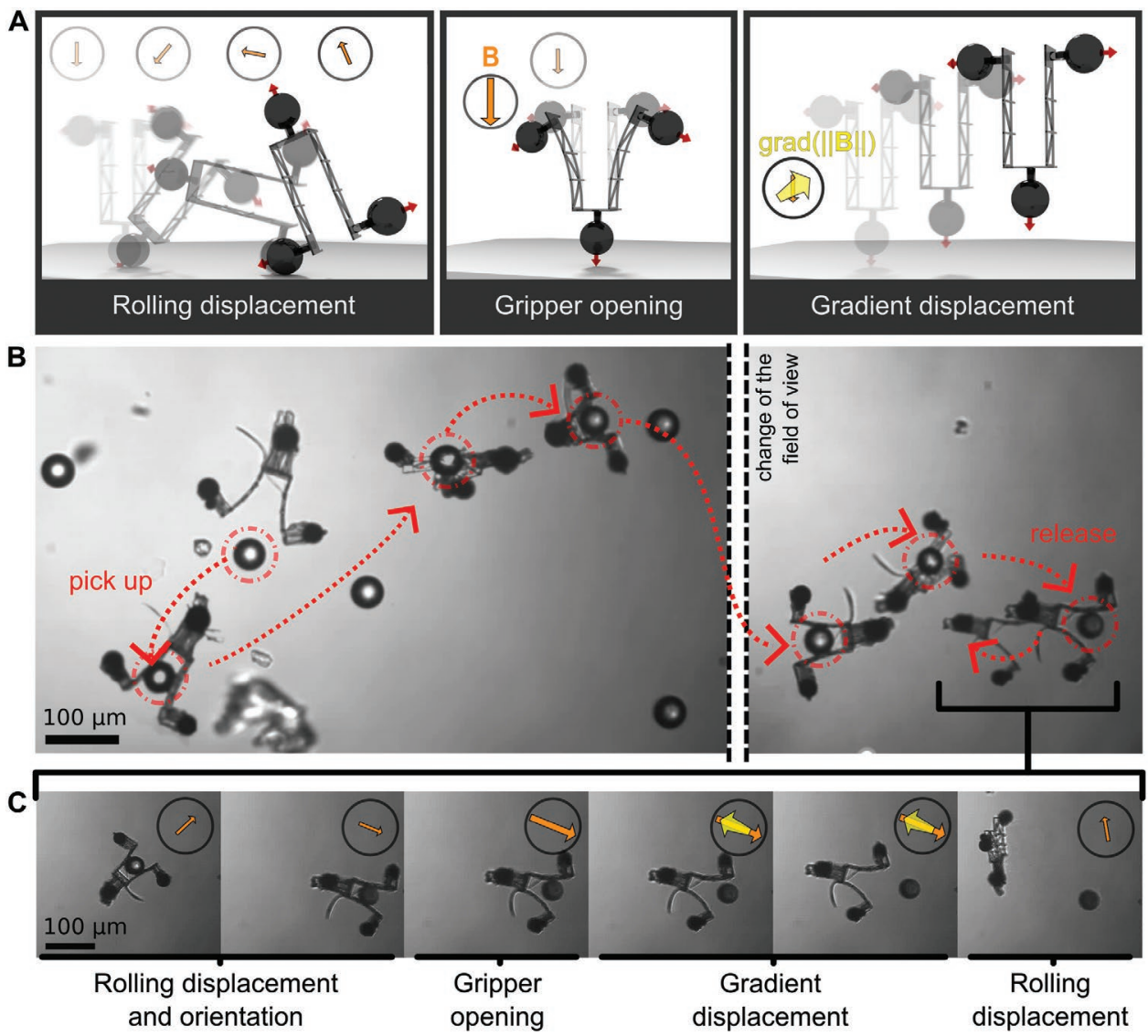

Figure 4. Magnetic actuation of the microgripper. A) Actuation modes of the microgripper. Left, rolling displacement: the central bead aligns along the external magnetic field as it exerts a magnetic torque on the bead. Center, gripper opening: a longitudinal magnetic field induces on the two lateral beads a magnetic torque, thus opening the gripper. The friction between the support and the gripper induces a rolling movement. Right, gradient displacement: subject to a magnetic field gradient, the gripper drifts smoothly along this gradient. B) Pick-and-place manipulation of polystyrene beads. The gripper is first opened to pick up a chosen $40 \mu \mathrm{m}$ polystyrene bead. The gripper is then brought by rolling motion to the desired place to release the bead, and moved away (Video S1, Supporting Information). C) Details of the release operation. The magnetic field is rotated to move and orient the microgripper. Increasing the magnetic field opens the gripper arms to release the bead. Next, a magnetic gradient is applied to gently move the microgripper away from the polystyrene bead. Lastly, the magnetic field is decreased to stop the gripper movement.

versatile microactuators. The complete independence between the geometry and magnetization allows for additional degrees of control through an external magnetic field. This new fabrication technique, which preserve the transparency of the polymer, was applied to develop the first magnetically actuable microlens. Furthermore, using the possibility of tailoring the magnetization direction in the microstructure, a microgripper was also fabricated. We demonstrated that even at a scale below $100 \mu \mathrm{m}$, such magnetic microactuators can be remotely and precisely actuated in translation, rotation, and deformation. In particular, the achieved dexterity was evidenced on the manipulation of objects which are comparable in size to some biological cells, opening considerable prospects in the field of life sciences.

\section{Experimental Section}

Materials: The photoresist used was an Ormocomp-based mixture prepared as a solution of $10 \mathrm{mg}$ of 1,3,5-tris (2-(9-ethylcabazyl-3) ethylene) benzene photoinitiator dissolved in $1 \mathrm{~mL}$ dichloromethane, and mixed with $1 \mathrm{~g}$ of OrmoComp.
For the experiment presented in Video S2 (Supporting Information), a hydrogel photoresist ${ }^{[36]}$ made by mixing $1 \mathrm{~mL}$ of deionized water with $200 \mathrm{mg}$ of gelatin (gelatin from bovine skin, Sigma-Aldrich), $30 \mathrm{mg}$ of collagen (collagen from rat tail, Sigma-Aldrich), and $10 \mathrm{mg}$ of dichlorotris (1,10-phenantroline) ruthenium(II) (Sigma-Aldrich) was used.

The magnetic beads that were used to make these hybrid structures were gas-atomized spherical $\mathrm{NdFeB}$ polycrystalline powders (MQP-S-11-9-20001, Magnequench) diverted from their initial use (industrial manufacturing of bonded magnets). Due to the broad size distribution of the commercial powders, the powders were sieved to limit the size of the beads between 20 and $30 \mu \mathrm{m}$.

Equipment: For structure fabrication, a Microlight two-photon polymerization 3D microprinter, combined with either a Zeiss or an Olympus inverted microscope with a $100 \times / 1.25$ objective lens, was used. The laser used for the photopolymerization was a pulsed $\mathrm{Nd}$-doped yttrium aluminium garnet laser $(\lambda=532 \mathrm{~nm}$, green). The scanning electron microscopy (SEM) images were acquired using a ZEISS Ultra+ SEM, after having coated the objects with a $15 \mathrm{~nm}$ thick gold layer.

Softwares: The models were designed with SolidEdge, Blender, and/ or FreeCAD, and exported to STL files. In order to print the structures, another file describing the polymerization dots needed to be created. This is called the slicing and was performed with Simpoly, and the printing was executed by Lithos (both by Microlight). The softwares used for the optical images' acquisition were $\mu$ Eye Cockpit for black and white 
and AVT SmartView 1.14 for color images, respectively. For simulations, COMSOL Multiphysics 5.4 was used. The figures were made using InkScape 0.92.1, Power Point 2016, GIMP 2.10, and Blender 2.79. The 3D animations and the videos were made using Blender 2.79 and Kdenlive 18.12 .

Beads' Magnetization: The beads were originally not magnetized. To magnetize them, a NdFeB ring magnet $(\varnothing 20.0 \times 4.0 \times 7.0 \mathrm{~mm}, \mathrm{~N} 35)$ was brought in contact with the backside of the glass slide, producing an external magnetic field of $500 \mathrm{mT}$ in the sample area. This applied field magnetized the beads with a remanence of $60 \mathrm{kA} \mathrm{m}^{-1}$ in the absence of an external magnetic field. The beads' remanent magnetization curve was measured and plotted with a vibrating-sample magnetometer (VSM) (Figure S3, Supporting Information). The sample consisted of magnetic beads dispersed in a solid nonmagnetic matrix (polydimethylsiloxane), and then cut up to fit in the VSM.

Design: The structures were first drawn and designed digitally, then their behavior and deformation were simulated using finite elements. For the simulations, the Young's modulus of the Ormocomp was chosen as $2 \mathrm{GPa}$, and the remanent magnetization of the beads as $60 \mathrm{kA} \mathrm{m}^{-1}$ ). These simulations were done prior to fabrication to approximately ensure adequate structure behavior, before printing the objects and testing them. If need be, the different parameters, such as thickness of the hinges or slicing parameters, were modified and tested again. A good correlation was observed between the experimental and simulated data (Figures S4 and S5, Supporting Information) but for more accuracy, calibration of the final system was still needed.

Microprinting: First, the 3D model was sliced with the following parameters: height of the voxel $=0.7 \mu \mathrm{m}$, width of the voxel $=0.3 \mu \mathrm{m}$, and overlap $=60 \%$. Then, the beads were sprinkled on a thin glass cover slip by hand with a plastic tip to get scattered beads on the glass slip, and these were covered with a drop of Ormocomp mixture. The beads should be spaced enough to have room to print but not too much that it is difficult to gather the beads for multibead objects. For the multibead structures, the beads were magnetized at this stage.

Next, the coverslip was observed under a microscope to find an appropriate place to print the model, near a bead for one-bead structure, and in the center of a group of beads for multibead objects. Then the focus plane was set to the interface between the glass and the photoresist. For one-bead structures, the sequential production of several objects was automated over many hours. For this purpose, the coordinates of every bead were input into the software as a reference for each structure. The parameters used for the laser were exposure time $=1 \mathrm{~ms}$ and voltage power $=0,14 \mathrm{~V}$.

Bonding the Beads: For one-bead structures, the bead was already placed since the structure was printed next to it. For multibead structures, the beads were moved through magnetic actuation; since the beads were magnetized, using a hand-held NdFeB ring magnet, they could be moved to the desired spot. First one bead was chosen to place, and it was moved to where it needs to be; it was oriented with the correct orientation; and then it was bonded to the printed structure. Now it would not move anymore, and the other beads could be attended and the same process could be repeated.

The link was a straight line printed from the object to the bead, to make a strong bond and minimize the production of bubbles (laser parameters: exposure time $=10 \mathrm{~ms}$, voltage power $=0.22 \mathrm{~V}$ ). The precision of the positioning of the beads by rotation/friction was around $2 \mu \mathrm{m}$.

Washing: The hybrid structures were washed in acetone. First, the coverslip was submerged in acetone for $10 \mathrm{~min}$, upside down with a tilt angle to avoid crushing the structure between the bottom of the Petri dish and the cover slip. The cover slip with the structures was then dried slowly under a glass Petri dish with a few drops of acetone around it to limit the effect of capillary forces on the printed structures during evaporation.

Actuation: One-bead structures were magnetized before actuation, by applying a magnet to the bottom of the cover slip. All structures were actuated with hand-held $\mathrm{NdFeB}$ magnets, and observed under the same microscopes as those used for structure fabrication. When needed, the structures were mechanically detached with a second coverslip or a tungsten needle, both by hand and under the microscope. The structures were either actuated in air (for optical lenses) or in water mixed with 5 wt\% Tween 20 to avoid adhesion to the glass slip.

Optical Experiment: For imaging the microlens, an objective lens, Olympus $40 \times 10.95$, was used. The microlens is a biconvex lens with both radii of curvature of $20 \mu \mathrm{m}$ and a refractive index of $n=1.52$. The focal length for a biconvex lens is defined as (Lens Maker equation)

$\frac{1}{f}=(n-1)\left(\frac{1}{r_{1}}-\frac{1}{r_{2}}+\frac{n-1}{n} \times \frac{t}{r_{1} r_{2}}\right)$

In this case, the obtained focal length was

$\frac{1}{f}=0.52\left(\frac{1}{20}-\frac{1}{20}+\frac{0.52}{1.52} \times \frac{5.79}{400}\right)$

$f=18.3 \mu \mathrm{m}$

This was confirmed experimentally (Figure S4, Supporting Information).

Pick-and-Place Experiment: $40 \mu \mathrm{m}$ polystyrene beads (Dynoseeds TS40, by Microbeads) dispersed in a water-based solution were used.

Mechanical Characterization of the Photoresist: Nanoindentation was used to measure locally the resin's elastic property on a microprinted pad ( $5 \mu \mathrm{m}$ high, $20 \mu \mathrm{m}$ large), giving a Young's modulus of $2 \mathrm{GPa}$ later used for simulations. Indentation modulus $\left(E /\left(1-v^{2}\right)\right.$, with $E$ and $v$ being the Young's modulus and Poisson ratio, respectively) was obtained using the standard method of Oliver and Pharr ${ }^{[37]}$ with continuous stiffness measurements ( $2 \mathrm{~nm}$ oscillation at $45 \mathrm{~Hz}$ ) with a Berkovich diamond tip and a strain rate of $0.05 \mathrm{~s}^{-1}$ on an MTS-XP machine.

Adhesive Strength Measurement: The adhesive strength of the bond was estimated thanks to a micromechanical structure (Figure S2, Supporting Information) composed of a magnetic bead attached on one side to the end of a microprinted deflecting beam through one bond (the test bond that will be broken) and on its other side to a microprinted pulling ring through three bonds (so as not to break up during the test). The base of the deflection beam was fixed to the support to allow the deflection of the beam when the bead was pulled perpendicularly to the beam. Its deflection was measured optically during the test (Olympus inverted microscope with a $20 \times / 0.4$ objective lens). The pulling ring was initially rested on thin pillars fixed on the substrate. During the traction experiment, a needle mounted on a microactuator (Siskiyou MX630L) was inserted into the ring to pull it perpendicularly to the beam until the single bond was broken. The maximum deflecting distance (just before bond failure) was measured on three samples $(3.9 \pm 0.1 \mu \mathrm{m})$ and used to deduce the corresponding adhesive force of $\approx 50 \mu \mathrm{N}$. For the force calculation, the transverse stiffness was approximated to $14 \mathrm{~N} \mathrm{~m}^{-1}$.

A numerical simulation of the transverse stiffness gave the value of $13.7 \mathrm{~N} \mathrm{~m}^{-1}$. The order of magnitude of the simulation was validated by comparing the vertical stiffness obtained by simulation $\left(14.8 \mathrm{~N} \mathrm{~m}^{-1}\right)$ to the experimental one obtained from force spectroscopy measurement on five samples $\left(15.4 \pm 1.6 \mathrm{~N} \mathrm{~m}^{-1}\right)$. Force spectroscopy was performed with a JPK BioAFM with an HQ-150-Au probe (probe stiffness $=14.9 \mathrm{~N} \mathrm{~m}^{-1}$ determined by the thermal noise method), by pressing on the end of the microprinted beam to induce a deflection of around $1 \mu \mathrm{m}$. The bonding surface estimated from SEM images was about $10 \mu \mathrm{m}^{2}$, and the bonding stress was then $\approx 5 \mathrm{MPa}$.

\section{Supporting Information}

Supporting Information is available from the Wiley Online Library or from the author. 


\section{Acknowledgements}

The authors thank Microlight3D for discussions and free assistance with the microprinter, N. M. Dempsey for discussions on the manuscript, V. Balcato and S. Le Denmat for assistance with atomic force microscopy, J.-F. Motte for assistance with SEM, L. Gredy for photoresist fabrication, and A. Mery and P. Marmottant for stimulating discussions. This work was performed between G2Elab, LIPhy and Institut Néel. This work was funded by a Ph.D. scholarship from Institut Carnot/Energies du Futur.

\section{Conflict of Interest}

The authors declare no conflict of interest.

\section{Keywords}

hybrid materials, magnetic microactuators, micro-optics, microtweezers, two-photon polymerization

[1] S. Kawata, H.-B. Sun, T. Tanaka, K. Takada, Nature 2001, 412, 697.

[2] T. Gissibl, S. Thiele, A. Herkommer, H. Giessen, Nat. Photonics 2016, 10, 554.

[3] P.-I. Dietrich, M. Blaicher, I. Reuter, M. Billah, T. Hoose, A. Hofmann, C. Caer, R. Dangel, B. Offrein, U. Troppenz, M. Moehrle, W. Freude, C. Koos, Nat. Photonics 2018, 12, 241.

[4] F. Klein, T. Striebel, J. Fischer, Z. Jiang, C. M. Franz, G. von Freymann, M. Wegener, M. Bastmeyer, Adv. Mater. 2010, 22, 868.

[5] S. Kim, F. Qiu, S. Kim, A. Ghanbari, C. Moon, L. Zhang, B. J. Nelson, H. Choi, Adv. Mater. 2013, 25, 5863.

[6] S. E. Chung, X. Dong, M. Sitti, Lab Chip 2015, 15, 1667.

[7] R. L. Truby, J. A. Lewis, Nature 2016, 540, 371.

[8] X. Kuang, D. J. Roach, J. Wu, C. M. Hamel, Z. Ding, T. Wang, M. L. Dunn, H. J. Qi, Adv. Funct. Mater. 2019, 29, 1805290.

[9] S. Palagi, P. Fischer, Nat. Rev. Mater. 2018, 3, 113.

[10] L. Hines, K. Petersen, G. Z. Lum, M. Sitti, Adv. Mater. 2017, 29, 1603483.

[11] Y. Lin, J. Xu, Adv. Opt. Mater. 2018, 6, 1701359.
[12] X. Wang, X.-H. Qin, C. Hu, A. Terzopoulou, X.-Z. Chen, T.-Y. Huang, K. Maniura-Weber, S. Pané, B. J. Nelson, Adv. Funct. Mater. 2018, 28, 1804107.

[13] T.-Y. Huang, M. S. Sakar, A. Mao, A. J. Petruska, F. Qiu, X.-B. Chen, S. Kennedy, D. Mooney, B. J. Nelson, Adv. Mater. 2015, 27, 6644.

[14] S. Tottori, L. Zhang, F. Qiu, K. K. Krawczyk, A. Franco-Obregón, B. J. Nelson, Adv. Mater. 2012, 24, 811.

[15] Y. Tian, X. Shao, J. Nanomater. 2016, 2016, 9781601.

[16] C. Peters, O. Ergeneman, P. D. W. García, M. Müller, S. Pané, B. J. Nelson, C. Hierold, Adv. Funct. Mater. 2014, 24, 5269.

[17] H. Xia, J. Wang, Y. Tian, Q.-D. Chen, X.-B. Du, Y.-L. Zhang, Y. He, H.-B. Sun, Adv. Mater. 2010, 22, 3204.

[18] X.-Z. Chen, M. Hoop, F. Mushtaq, E. Siringil, C. Hu, B. J. Nelson, S. Pané, Appl. Mater. Today 2017, 9, 37.

[19] Z. Li, E. Diller, Int. J. Adv. Rob. Syst. 2017, 14, 1729881416682707.

[20] C. Hu, S. Pané, B. J. Nelson, Annu. Rev. Control Rob. Auton. Syst. 2018, 1, 53 .

[21] C. de Marco, S. Pané, B. J. Nelson, Sci. Rob. 2018, 3, eaau0449.

[22] E. Diller, M. Sitti, Adv. Funct. Mater. 2014, 24, 4397.

[23] T. Qiu, T.-C. Lee, A. G. Mark, K. I. Morozov, R. Münster, O. Mierka, S. Turek, A. M. Leshansky, P. Fischer, Nat. Commun. 2014, 5, 5119.

[24] W. Hu, G. Z. Lum, M. Mastrangeli, M. Sitti, Nature 2018, 554, 81.

[25] Y. Kim, H. Yuk, R. Zhao, S. A. Chester, X. Zhao, Nature 2018, 558, 274.

[26] J. Kim, S. E. Chung, S.-E. Choi, H. Lee, J. Kim, S. Kwon, Nat. Mater. 2011, 10, 747.

[27] C. Peters, M. Hoop, S. Pané, B. J. Nelson, C. Hierold, Adv. Mater. 2016, 28, 533.

[28] C. de Marco, C. C. J. Alcântara, S. Kim, F. Briatico, A. Kadioglu, G. de Bernardis, X. Chen, C. Marano, B. J. Nelson, S. Pané, Adv. Mater. Technol. 2019, 4, 1900332.

[29] M. A. Zeeshan, R. Grisch, E. Pellicer, K. M. Sivaraman, K. E. Peyer, J. Sort, B. Özkale, M. S. Sakar, B. J. Nelson, S. Pané, Small 2014, 10, 1284.

[30] C. C. J. Alcântara, S. Kim, S. Lee, B. Jang, P. Thakolkaran, J. Kim, H. Choi, B. J. Nelson, S. Pané, Small 2019, 15, 1805006.

[31] S. Li, J. Qin, A. Fornara, M. Toprak, M. Muhammed, D. K. Kim, Nanotechnology 2009, 20, 185607.

[32] R. M. Erb, J. J. Martin, R. Soheilian, C. Pan, J. R. Barber, Adv. Funct. Mater. 2016, 26, 3859.

[33] J. Torgersen, X.-H. Qin, Z. Li, A. Ovsianikov, R. Liska, J. Stampfl, Adv. Funct. Mater. 2013, 23, 4542.

[34] T. Xu, J. Zhang, M. Salehizadeh, O. Onaizah, E. Diller, Sci. Rob. 2019, 4, eaav4494.

[35] J. Cui, T.-Y. Huang, Z. Luo, P. Testa, H. Gu, X.-Z. Chen, B. J. Nelson, L. J. Heyderman, Nature 2019, 575, 164.

[36] O. Stephan, L. Gredy, US20190113841A1, 2019.

[37] W. C. Oliver, G. M. Pharr, J. Mater. Res. 1992, 7, 1564. 